\title{
AFM-based 3D Nanofabrication using Ultrasonic Vibration Assisted Nanomachining
}

Jia Deng, Li Zhang, Jingyan Dong*, Paul H. Cohen

Edward P. Fitts Department of Industrial and Systems Engineering

North Carolina State University

Raleigh, North Carolina, USA

* Corresponding Author

Email: jdong@ncsu.edu

Tel: 919-515-7196

Fax: 919-515-5281

414-C Daniels Hall, Campus box 7906

Department of Industrial and Systems Engineering

North Carolina State University

Raleigh, North Carolina 27695-7906, USA 


\title{
AFM-based 3D Nanofabrication using Ultrasonic Vibration Assisted Nanomachining
}

\author{
Jia Deng, Li Zhang, Jingyan Dong and Paul H. Cohen \\ Edward P. Fitts Department of Industrial and Systems Engineering \\ North Carolina State University \\ Raleigh, North Carolina, USA
}

\begin{abstract}
This paper presents a novel AFM-based 3D nanofabrication process using ultrasonic vibration assisted nanomachining. A set of three dimensional nanostructures on polymethyl methacrylate (PMMA) samples are fabricated with the assistance of high frequency in-plane circular xy-vibration and ultrasonic tipsample z-vibration. Two methods for fabricating 3D nanostructures were investigated in this study, which are layer-by-layer nanomachining and one pass nanomachining with the depth controlled by setpoint force. Critical parameters in the process are identified, including setpoint force, overlap percentage, amplitude of $\mathrm{z}$ vibration and machining speed. By regulating these process parameters, multi-level 3D nanostructures were fabricated by multi-layer machining in vector mode and raster scan mode. Using different setpoint forces for regulating feature depths, other nanostructures, such as convex and concave circles, were fabricated in raster scan mode from gray-scale bitmap pattern images. Under each mode, 3D nanostructure over microscale area can be fabricated in just a few minutes with sub-10 nm resolution in $\mathrm{z}$ direction.
\end{abstract}

Keywords: 3D nanomachining, Atomic force microscope (AFM), Tip-based nanofabrication, Ultrasonic vibration assisted nanomachining

\section{Introduction}

Nanotechnology has significant impacts on many fundamental research areas such as physics, chemistry and biology, as well as the modern electronic devices that have reached nanoscale domain such as optoelectronic devices. There are many advanced nanofabrication techniques such as nanomanipulation [1], nanopatterning [2], nanomachining [3], nanosurgery [4], and nanodissection [5]. Among these techniques, tip-based nanomachining [6-8] as a low-cost and easy-to-setup nanofabrication technique has been implemented as a direct modification tool to modify the surface of the sample mechanically to produce sub-50nm patterns on different types of surfaces such as polymers, silicon and metals [9-16]. In tip-based nanomachining, Scanning Probe Microscopy (SPM), including Scanning Tunneling Microscopy (STM) and Atomic Force Microscope (AFM), can be used both as an image tool and modification tool 
[17], which provide the in-situ visualization and inspection capabilities of nanomachining. However, it is very challenging for traditional tip-based nanomachining to achieve high-rate fabrication of nanostructures. The widely used direct mechanical scratching methods are limited in the machining speed due to the large interaction force required between tip and surface of sample. The feature created by direct mechanical scratching generally has a very small depth and width, which is inefficient to form nanostructures at large scale, especially for 3D nanostructures with relatively large depth.

Many approaches for the fabrication of 3D nanostructures were developed, such as e-beam lithography [18], laser nanopatterning [19-20], focused ion beam (FIB) milling [21], UV nanoimprint lithography [22], colloidal lithography [23], anisotropic etching [24] and corner lithography [25] etc. E-beam lithography is capable to fabricate sub-100 nm nanostructures [18], but the process of creating 3D structure needs the carefully planned sample rotation and controlled beam positioning/dosage. The complexity in the process limits the variety of nanostructures, precision and speed of fabrication. In laser nanopatterning, feature sizes are limited by wavelength and spot size of laser beam. Generally laser sources for nanolithography are costly, have low output power and unstable light intensity [19]. Femtosecond pulsed laser machining can be used to make lines with $500 \mathrm{~nm}$ width on copper surfaces and $800 \mathrm{~nm}$ width on stainless steel surfaces. However, features with less than $100 \mathrm{~nm}$ sizes are extremely difficult to be created with the pulsed laser beam, which has hundreds of nm in wavelength, which make this technique mostly suited for the fabrication of sub-micrometer structures other than nanometer scale structures [20]. FIB milling process uses ion-bombardment to create surface patterns step by step to achieve 3D structures [21]. Redeposition during FIB milling process determines could potentially contaminate the sample. Similar to e-beam lithography and laser nanopatterning, FIB process also relies on specific equations to provide the energy beam for nanomachining, which make it expensive to use and maintain.

Tip-based nanomachining processes have unique advantages over e-beam lithography, laser nanopatterning and many other lithography processes, as they are low-cost, low-effort processes, and have greater potential to fabricate complex 3D nanostructures. Since AFM is originally used as a highresolution imaging and metrology tool, inspecting the original surfaces and visualizing final nanostructures before and after machining are naturally included in AFM-based machining. As a result, it is relatively easy to align features on the previous machined surface towards a complex 3D structure. There are, however, some limitations including the large setpoint force and small material removal rate, which results in high tip wear rate, slow machining speed and low throughput. In our previous study, an AFM-based high-rate tunable ultrasonic vibration assisted nanomachining process was developed [26-27] for high speed nanofabrication on PMMA and aluminum samples. Utilizing this process, 3D 
nanostructures can be fabricated in high speed by designing the corresponding machining procedure and tuning the input parameters properly.

In this paper, a 3D nanofabrication process is developed using the AFM-based ultrasonic vibration assisted nanomachining process. Two pattern generation methods were utilized in this newly developed process, which are vector mode based pattern generation and raster scan mode based pattern generation. Multi-level 3D stair-like nanostructures were fabricated in both vector mode and raster scan mode, while in vector mode, the multi-layer nanostructures were fabricated by layer-by-layer nanomachining technique, and in raster scan mode, the nanostructures were fabricated in one pass with the feature depth regulated by the force specified by the gray-scale bitmap image. Other complex nanostructures, such as convex and concave circles, were fabricated similarly in raster scan mode. Critical process parameters were identified for the $3 \mathrm{D}$ nanofabrication process, which include the setpoint force, the overlap percentage, amplitude of $\mathrm{z}$ vibration, and machining speed. These parameters in the process were tuned carefully to achieve the desired feature dimension and process performance. The experimental fabrication results demonstrate the 3D fabrication capability of the AFM-based ultrasonic vibration assisted nanomachining process.

\section{Experimental Setup and Process Design}

\subsection{Experimental Setup}

The experimental nanomachining system includes a commercial AFM (XE-70 from Park Systems) and a lab-designed nanovibrator stage, as shown in Figure 1 (a). The pillar at the center of the nanovibrator stage is actuated by two xy-piezo actuators, generating high frequency (2 kHz) in plane xyvibration. Another z-piezo actuator is attached on the top of the pillar, providing ultrasonic vibration (2 $\mathrm{MHz}$ ) to the sample attached on the top of the z-piezo actuator. All of the piezo actuators in xy and $\mathrm{z}$ direction are driven by sinusoid signals with appropriate voltages, generated from a commercial signal output device (USB-6259) from National Instrument. Interaction force between the AFM tip and the sample surfaces is measured by the optical system and photo detector from the AFM. The force signals are acquired into computer to regulate the setpoint force and to monitor the process.

In the ultrasonic vibration assisted nanomachining process, two piezoelectric actuators in $\mathrm{x}$ and $\mathrm{y}$ directions are driven by sinusoid signals with 90-degree phase difference to generate a circular motion. With the assistance of in-plane circular xy-vibration, a virtual tool is formed, which is able to control the feature width in one machining pass. As shown in Figure 1 (c), assuming semi-spherical tip geometry, the outer edge of the circular path can be viewed as a virtual tool with a single cutting tooth that is engaged in material removal. The diameter of the virtual tool is the summation of the diameter of the tip and the 

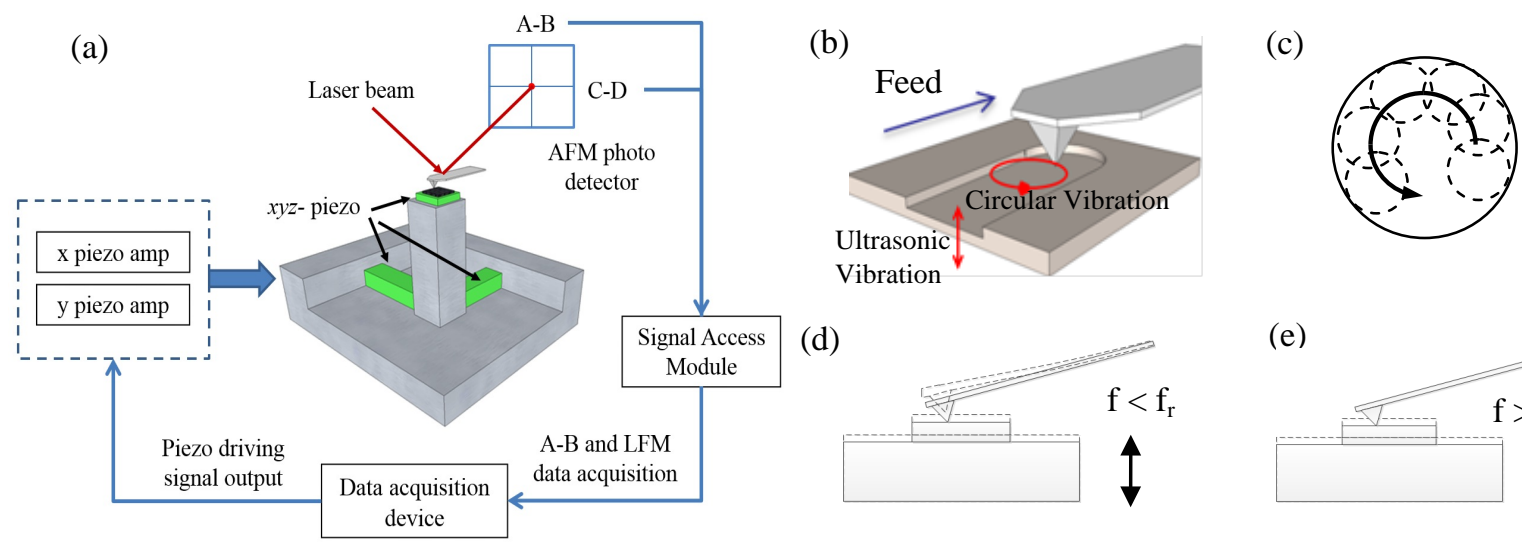

(d)

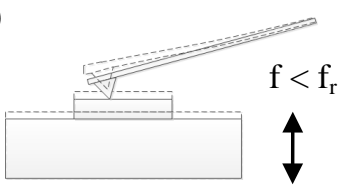

(e)

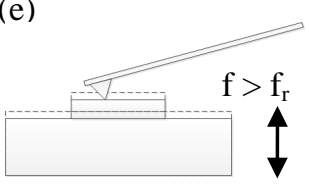

Figure 1: (a) Schematic illustration of nanovibrator in the experimental setup. (b) Schematic illustration of nanomachining assisted by vibration. (c) Virtual tool when tip is vibrated with a xy circular path. (d), (e) Tipsample interaction with z-vibration in low frequency and high frequency.

amplitude of the circular motion, which can be experimentally characterized. For example, when the driving voltage of xy-vibration is $30 \mathrm{mV}$, the diameter of virtual tool is around $100 \mathrm{~nm}$ at the trench depth of $10 \mathrm{~nm}$. The diameter of the virtual tool is the sum of tip diameter, which is $30 \mathrm{~nm}$, and the diameter produced by xy-vibration, which is $70 \mathrm{~nm}$ at the vibrational amplitude of $30 \mathrm{mV}$. The diameter of the virtual tool is controlled by the amplitude of the xy vibration, which directly regulates the feature width that can be machined in one machining pass. With the tip rotation, a thin slice of material (defined as feed per rotation) is removed in one machining cycle, which significantly reduces machining force between the tip and the sample and increases the machining speed [26, 27]. By choosing different feeds, the load/force during each cycle can be regulated. Besides the xy-vibration, the ultrasonic z-vibration between the tip and the sample helps control the depth during machining process. When the z-vibrator drives samples at a frequency that is less than the resonant frequency of the cantilever $\left(f<f_{r}\right)$, the cantilever, contacting with the sample surface, follows the vibration of samples, as shown in Figure 1 (d). However, when the zvibrator drives samples at a frequency that is much higher than the resonant frequency of the cantilever ( $\mathrm{f}$ $>>f_{r}$ ), the cantilever dynamically 'freezes' due to its inertia, and immerses into sample surfaces for effective nanomachining, as shown in Figure 1 (e) [26-27]. In our nanomachining process, the frequency of z-vibration is selected to be $2 \mathrm{MHz}$, which is much higher than the resonant frequency of the cantilever beam of $190 \mathrm{kHz}$.

In this study, a tapping mode silicon probe with a diamond like carbon (DLC) coating is used in nanomachining with dimension of $225 \mu \mathrm{m} \times 38 \mu \mathrm{m} \times 7 \mu \mathrm{m}$. The nominal stiffness of the cantilever is 48 $\mathrm{N} / \mathrm{m}$ and the resonant frequency is $190 \mathrm{kHz}$. Images are taken immediately after the machining process. Tips used in the machining experiments are also used in imaging before and after nanomachining. The nanomachining process was applied on polymethyl methacrylate (PMMA) film. The PMMA (950PMMA A4 from Microchem, 4\% dilution in anisole) was spin-coated on a cleaned silicon substrate for $40 \mathrm{~s}$ at 
4000 RPM to get a film with thickness of $200 \mathrm{~nm}$, and then the wafers were baked at $180{ }^{\circ} \mathrm{C}$ for $90 \mathrm{~s}$. All experiments were performed at room temperature in air.

\subsection{Process Design for 3D Nanofabrication}

The 3D nanomachining approach in this study is developed by integrating ultrasonic vibration assisted nanomachining process [26-27] with the proper design of feature patterns and machining procedures. The AFM is generally used as a metrology tool for nanoscale imaging. However, when used for nanolithography, the AFM first scans an image on the selected area as the workspace for future lithography tasks, and nanolithography functions can be activated for feature fabrication by choosing different working modes, designing objects, selecting force values and machining speed. Depth control is the most critical part in 3D nanofabrication. In nanolithography using an AFM, there are two ways to control the tip-sample contact and the resulting feature depth, which are the constant z-height mode and the setpoint mode. Constant z-height mode is an open-loop control mode, in which the cantilever is moved to a specific z-height during the machining process. The setpoint force mode is a close-loop control mode, in which the controller keeps adjusting the cantilever position to maintain the desired contact force (reflected by the cantilever deflection) between the tip and the sample. Generally, the sample is not perfectly flat and the sample surface may not be parallel to the xy-plane of the AFM scanner. As the result, using constant height mode in nanomachining may incur undesired varying load during the machining process and degrade the machining performance. Therefore, in this study, the setpoint force mode is chosen to control the machining depth. By varying the setpoint force applied on PMMA samples, different depths can be achieved. Before the machining process, a calibration procedure is performed to identify the required force to obtain the desired feature depth under a certain overlap rate, amplitude of vibrations and the machining speed. The calibration process is implemented by machining a few trial features under a set of setpoint forces and measure the depths from them. After that, linear interpolation is used to set setpoint force for 3D nanofabrication processes.

Nanolithography functions provided by the Park Systems AFM were used to design nanomachining procedures for 3D fabrication. Two categories of feature objects can be designed in the nanolithography software: vector objects and bitmap images. The two pattern design methods indicate two working modes that are different in controlling the tip motion (similar to toolpath in conventional computer numerical control machining): vector mode and raster scan mode, as shown in Figure 2 (a) and (c).

In the vector mode, a set of basic shapes can be selected and combined together to construct the desired patterns. These basic shapes include point, line, rectangle, ellipse, polygon and polyline. By carefully arranging these basic shapes in sequence, both 2D and 3D patterns can be designed, as shown in Figure 2 (a). Some examples of vector-based toolpaths and the resulting 2D features and a 3D 
(a)

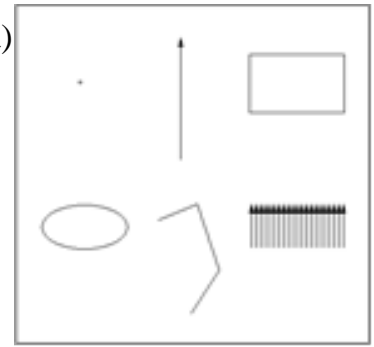

(b)

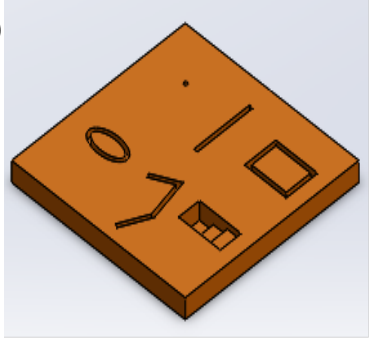

(c)

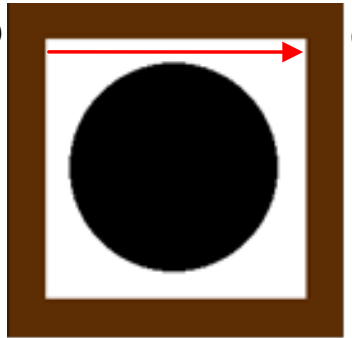

(d)

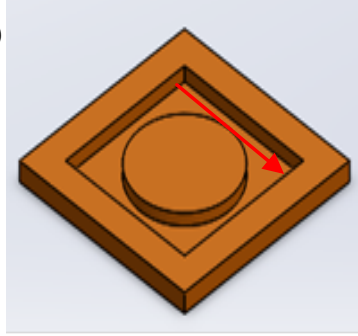

Figure 2: (a) Shapes designed in vector mode with an example of a three-layer 3D nanostructure. (b) Features after fabrication in vector mode. (c) Schematic of a 3D nanostructure in raster scan mode with a protruding circle represented by gray-scale bitmap image. Red line shows the scan direction. (d) Feature after fabrication in raster scan mode. Red line shows the scan direction.

nanostructure with three height levels after fabrication are illustrated in Figure 2 (b). In our experiments of fabricating the multi-level stair-like nanostructures, a number of parallel lines are designed for each layer to remove material at each specific height level. Lines are arranged from the left to the right and are distributed uniformly. The distance between two adjacent lines needs to be selected by considering the width of trench resulting from each line (from virtual tool diameter) and the overlap percentage between adjacent lines. The width of feature of each line is determined by the diameter of the virtual tool, which is characterized experimentally. Overlap percentage is related to the radial depth of cut in conventional scale machining, and will affect the surface finish of the final features. A large overlap percentage indicates a small radial depth of cut, which in general produces smoother part surface, but takes longer to finish the whole part. In our experiments, a 50\% overlap percentage was selected to achieve reasonable surface finish around $1 \mathrm{~nm}$, which is the surface finish of the PMMA film after spinning coating. A relatively large overlap percentage will not degrade the surface finish. The diameter of virtual tool is experimentally characterized to be around $100 \mathrm{~nm}$ when the driving voltage of xy-vibration is $30 \mathrm{mV}$. As a result, the distance between every two adjacent lines is $50 \mathrm{~nm}$. During the nanomachining process, AFM tip follows the pre-designed lines one by one according to the designed sequence. The setpoint force used for machining is around 150 to $300 \mathrm{nN}$, depending on the designed feature depth. When the tip makes a transition between lines, the interaction force applied on the sample changes to the background force (1 $\mathrm{nN}$ ). Besides the overlap percentage and setpoint force, the machining speed and amplitude of z-vibration also affect the fabrication results of the nanostructure. In this study, $1 \mu \mathrm{m} / \mathrm{s}$ machining speed is chosen, which corresponds to $0.5 \mathrm{~nm}$ feed per rotation. $3 \mathrm{~V}$ driving voltage is applied on z-piezo to provide $\mathrm{z}$ vibration to facilitate material removal.

In the raster scan mode, a gray-scale bitmap image is used by the nanolithography software for structure design. The gray-scale of the bitmap image corresponds to setpoint force at each pixel. The brightest color (pure white) and the darkest color (pure black) in the bitmap image represent the selected maximum and minimum setpoint force respectively, and the setpoint force is linearly interpolated by the 
gray-scales. The tip scans the bitmap image pixel-by-pixel from left to right and line by line from the top to the bottom, as shown in Figure 2 (c). The number of the rows and columns in the bitmap image and the overall size of the bitmap image determine the machining resolution. The number of pixel rows in the image is the number of traces the AFM tip scans laterally, which also determines the overlap percentage between two adjacent machining paths. The pixels in each row determine the positioning resolution, as the setpoint force is defined at each pixel. The laterally scanning direction is the only direction supported by this mode. Figure 2 (c) and (d) show a designed structure represented by the bitmap image and the resulting 3D nanostructure after fabrication. Compared with manually arranged toolpath in the vector mode, complex structures can be conveniently produced by simply loading pre-designed bitmap images into nanolithography software.

Under the vector mode and the raster scan mode, two techniques are developed for fabricating 3D nanostructures: layer-by-layer nanomachining and one pass nanomachining with depth regulated by the setpoint force. In layer-by-layer nanomachining, the entire nanostructure is fabricated by machining patterns layer-by-layer while changing the prescribed patterns for each layer. In our experiments, multiple layers of 3D stair-like nanostructures were machined by this method. For example, if a 3D stair-like nanostructure with three steps of same height is to be fabricated. A pocket with designed depth is fabricated as the first step. Then two third of the pocket is machined again with the same additional depth to create the middle step. Finally, the third step is fabricated by machining the one third area of the pocket with the same depth. During layer-by-layer machining process, the intermediate results after finishing each layer is able to be imaged to make sure the modification in the previous step has been finished successfully. The first layer of pattern can be designed with a certain number of parallel lines or a monocolored bitmap image (thus constant setpoint force) covering the desired area. The second and the third layer patterns were fabricated by covering only part of the pattern from previous steps. In one pass nanomachining with depth regulated by the setpoint force, the entire 3D pattern can be fabricated by one machining pass using the gray-scale image without any interruption during the machining process. The overall machining procedures are almost the same with layer-by-layer machining except that bitmap images are used in designing the pattern other than using basic simple geometric components to construct the patterns.

The overall 3D nanomachining procedure include: 1) Image the desired surface area under tapping mode by image software; 2) Change tapping mode to contact mode and approaching tip to the surface with background force $(1 \mathrm{nN})$; 3) Control the AFM with nanolithography software under setpoint force mode and designing desired patterns in the vector mode or the raster scan mode; 4) Apply xy and z 
vibration; 5) Start processes of machining; 6) Wash the sample with ultrasonic cleaning; and 7) Inspect the nanostructures after washing process.

After nanomachining, due to a large adhesion force at the nanometer scale, the debris and chips are not easily removed from the sample. A post-machining washing process was developed to remove the debris and chips around the machined structures. The washing procedures start from washing the sample with ultrasonic cleaning in 30\% wt aqueous solution of acetone for 2 minutes. Then the sample is washed by the DI water. Finally, the sample is washed with IPA solution and baked at $90{ }^{\circ} \mathrm{C}$ for 2 minutes. The concentration of the aqueous solution of acetone and the washing time has been characterized experimentally, so as not to damage the produced nanostructures.

\section{Results and Discussion}

In this study, the capability of AFM-based 3D nanomachining process was studied by implementing layer-by-layer nanomachining in both the vector mode and the raster scan mode, and one pass nanomachining in raster scan mode. These techniques in different modes provide us a variety of approaches to fabricate 3D nanostructures on PMMA films. The fabrication time of a typical 3D nanostructure with lateral dimension of $1.5 \mu \mathrm{m} \times 1.5 \mu \mathrm{m}$ is about 2-5 minutes in raster scan mode, which mainly depends on the nanomachining speed set by users. The dimensions of the successfully finished nanostructures has good repeatability when using the same input parameters.

\subsection{Nanomachining of 3D structures in the Vector Mode}

In the vector mode, 3D nanostructures can be produced by layer-by-layer nanomachining method, since it is not easy to reliably change the setpoint force during the vector-based toolpath. In this work, a three-level stair-like nanostructure was designed and fabricated in the vector mode. The overall size of the nanostructure is $1.5 \mu \mathrm{m} \times 1 \mu \mathrm{m}$, which has three steps with the width of $0.5 \mu \mathrm{m}$ for each step. Three layers of patterns were designed to implement the fabrication process, each of which is composed by a set of parallel vector lines. The designed pattern contains 30 lines for the first layer, 20 lines for the second layer and 10 lines for the third layer, with the space of 50nm between adjacent lines on all layers. The fabrication procedures are shown as follows (Figure 3 (a)): 1) Machining the first layer with the selected set of parameters (the driving voltage of xy-vibration is $30 \mathrm{mV}$ with the resulting diameter of virtual tool is around 100nm); 2) after that, reimaging the sample surface; 3) then aligning and machining the second layer using the same machining parameters on the same area of the first layer; 4) repeat steps 1-3 until all the layers are finished. After machining each layer, samples can be reimaged to inspect the results. Figure 3 (b) shows machining toolpaths for all three layers of 3D "stair-like" nanostructure, which is a set of 
(a)

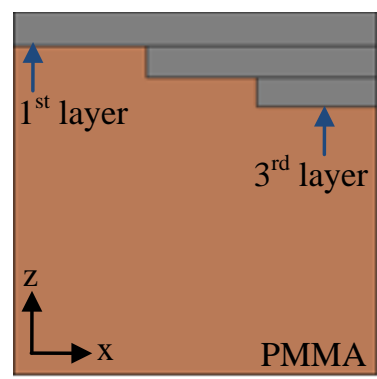

(d)

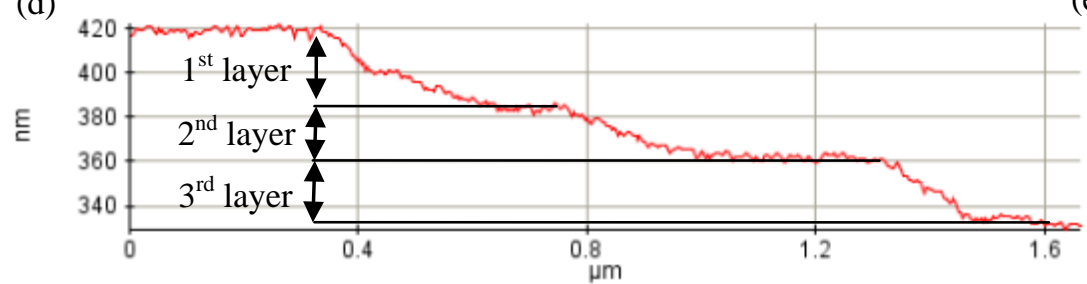

(c)

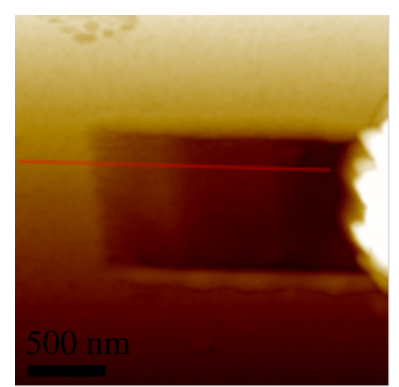

(e)

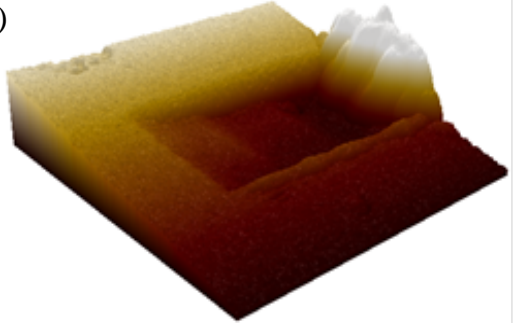

Figure 3 (a) Schematic of layer-by-layer based 3D nanofabrication process of a three-level nanostructure in vector mode. (b) Parallel vector lines (toolpaths) for all three layers of 3D "stair-like" nanostructure. (c) AFM image of the machined three-level nanostructure. (d) Cross sectional profile of the nanostructure. (e) 3D view of the three-level nanostructure.

parallel lines corresponding to the traces of AFM tip. The vectors on the second and third are 2/3 and 1/3 of the vectors from the first layer. The final result as shown in Figure 3 (c).

The setpoint force in this machining test was $150 \mathrm{nN}$ and the machining speed was $1 \mu \mathrm{m} / \mathrm{s}$. Driving voltage of xy-vibration and z-vibration are $30 \mathrm{mV}$ and $0.5 \mathrm{~V}$ respectively and the overlap percentage of adjacent lines is $50 \%$. The machined three-level stair nanostructure by layer-by-layer nanomachining is shown in Figure 3 (c) to (e). The result clearly shows three steps with the depths of each level at $35 \mathrm{~nm}$, 23nm and $27 \mathrm{~nm}$ respectively, as shown in Figure 3 (d). Depth of the first layer is slightly larger than the depths of the second and the third layer. The reason may be from the interaction of the debris and the AFM tip that prevent accurate depth achieved.

When using layer-by-layer machining in the vector mode to fabricate 3D nanostructures, overlap percentage is a critical factor to achieve the flat surface and good surface finish. When the overlap percentage is negative, gap between two machining paths is larger than the diameter of the virtual tool. A flat pocket surface cannot be achieved due to the un-machined areas between two paths. While the overlap percentage is too large ( $>60 \%$ ), the current machined feature is overlapped significantly with the previously machined features, and a wedge shape nanostructure will be produced instead of a flat structure at each layer. The reason comes from the fact that the depth is controlled by the setpoint force, not the absolute height of the tip. Using the same setpoint force, the feature depth can be affected by the different tip-sample interaction from the different overlap percentage of neighboring features. Other parameters also impact the machining result, including the amplitude of z-vibration and machining speed. 
Increasing the amplitude of z-vibration helps to increase the depth of trench for each machining layer, thus increase the dimension of final nanostructure in z direction. Increasing setpoint force has the similar effect to increase the feature depth [26, 27]. Decreasing the machining speed means decreasing the material feed to be machined for each in-plane vibration circle, which in turn increase the feature depth to some extent. The machining speed of $0.5 \mu \mathrm{m} / \mathrm{s}$ to $2 \mu \mathrm{m} / \mathrm{s}$ is a reasonable range observed in experiments.

Nanomachining using the vector mode has its advantages in its efficiency in designing and producing sparse patterns. Users can arrange vectors together as the toolpath to fabricate the desired patterns without scanning through all the areas as in the raster scan mode. However, it is relatively difficult to design complex 3D nanostructures in the vector mode, because all the vector components need to be manually placed in the workspace. For example, the stair-like structure can be designed easily by arranging vectorlines together, but a circle or other irregular features are very difficult to design, as they require many geometric calculations as well as manual placement of the vectors. Therefore, 3D nanofabrication process is investigated in raster scan mode using bitmap image for feature design.

\subsection{Nanomachining of 3D structures in the Raster Scan Mode}

In raster scan mode, the features are designed in the form of the gray-scale bitmap images with feature depths controlled by the gray-scale values, which represent different setpoint forces at each pixel. The amplitude of xy-vibration needs to be calibrated carefully to obtain a desired pixel dimension in the bitmap image. It is observed that the overlap percentage between two neighboring pixels need to be about $80 \%$ to obtain good machining uniformity, which is larger than the overlap percentage in the vector mode. The difference in the overlap percentage mainly comes from using different machining directions. In the raster scan mode, the cantilever is scanned in the lateral direction, which has a lower stiffness due to torsional motion of the cantilever, thus a large overlap percentage is required to produce the similar results from the vector mode, in which the machining direction is along the length direction of the cantilever.

The layer-by-layer machining is studied in the raster scan mode to fabricate 3D nanostructures. Figure 4 (a) shows the schematic of layer-by-layer machining process for a six-layer stair-like 3D nanostructure. For each layer, a mono-colored bitmap image (thus constant setpoint force) is used to remove material for that layer. Multiple machining processes are applied on the same aligned area to produce the designed 3D nanostructures. The shallowest layer that has been machined out is named as the first layer and so on. The overall size of the six-level nanostructure is $1.5 \mu \mathrm{m} \times 1.5 \mu \mathrm{m}$. Setpoint force is selected to be $150 \mathrm{nN}$ and the scanning speed to be $1 \mu \mathrm{m} / \mathrm{s}$. The driving voltages of xy-vibration and z-vibration are $30 \mathrm{mV}$ and $3 \mathrm{~V}$ respectively. As shown in the AFM image in Figure 4 (b) and (c), the 3D stair nanostructure with six height levels was successfully fabricated, after layer-by-layer machining and post-machining washing 
(a)

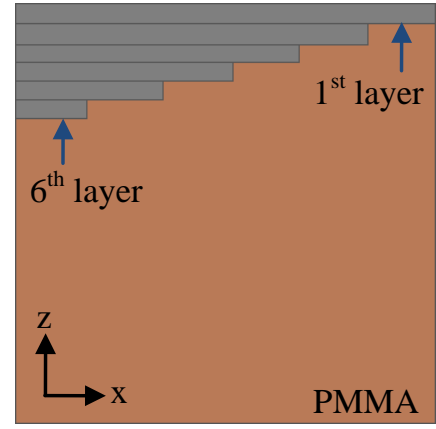

(b)

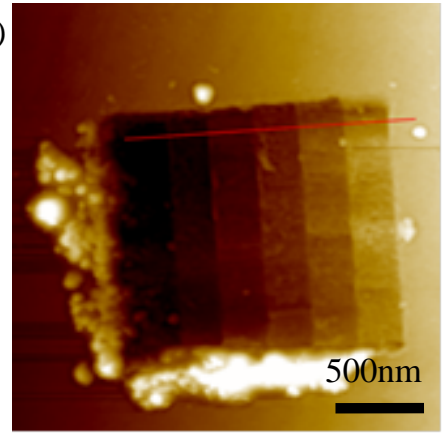

(c)

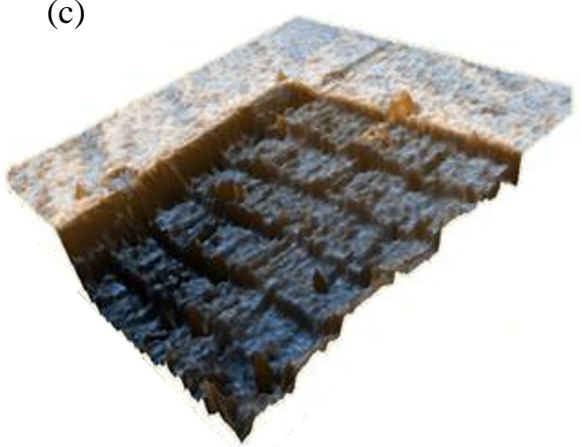

(d)

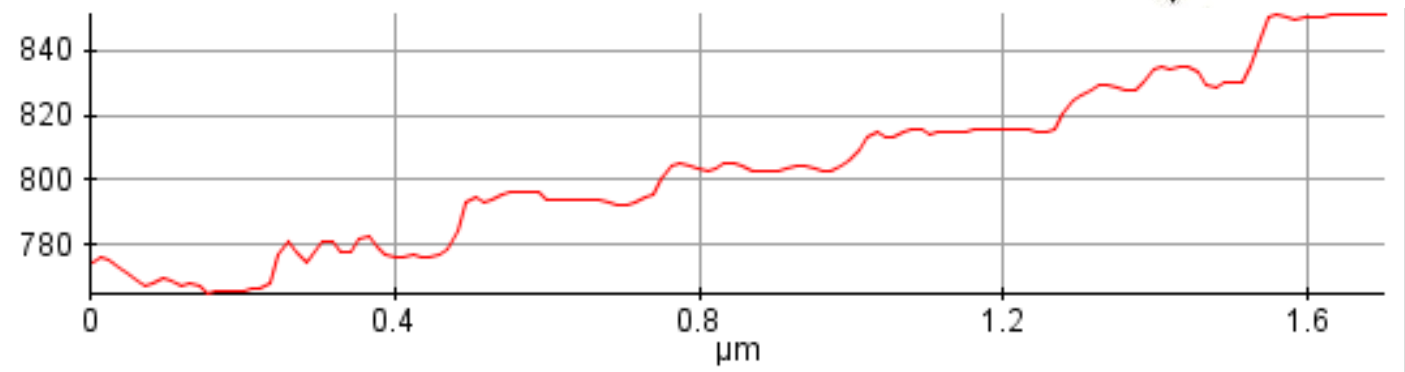

(e)

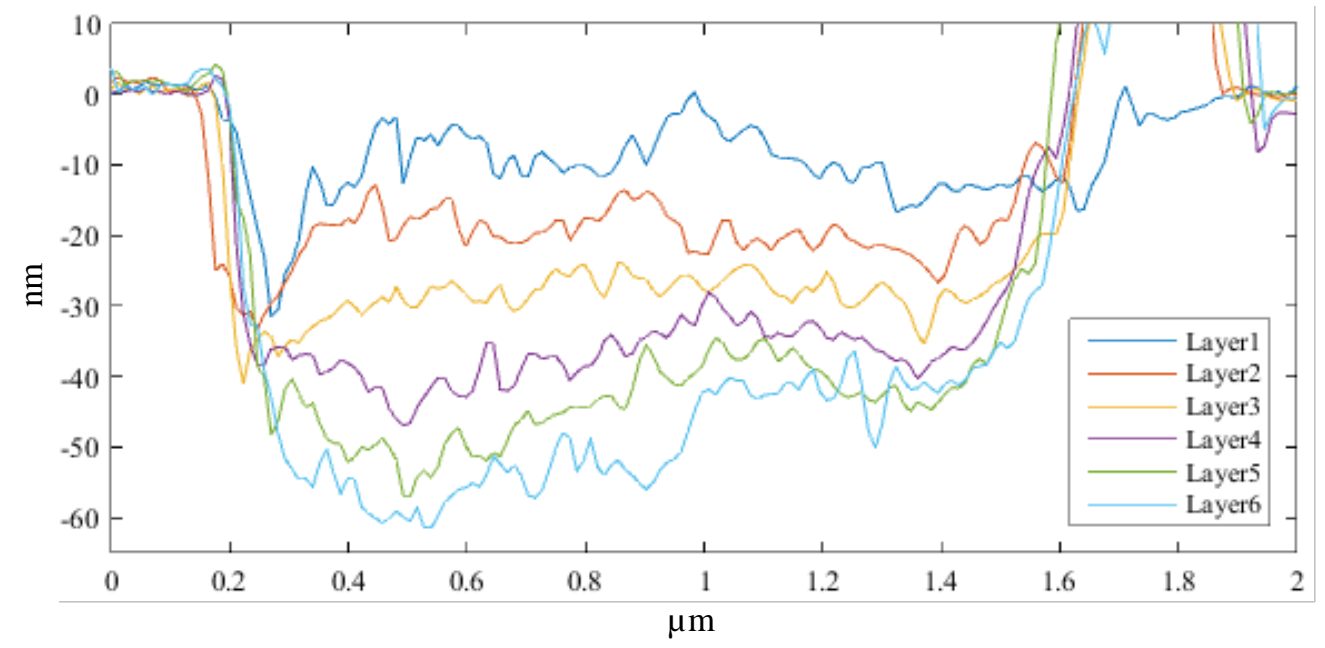

Figure 4: (a) Schematic of layer-by-layer based 3D fabrication process of a six layers nanostructure in the raster scan mode. (b) AFM image of the machined 3D nanostructure. (c) 3D view of the nanostructure. (d) Cross sectional profile of the feature clearly shows six height levels. (e) Cross-sectional profile of 6 layers.

process. From the cross section profile of the fabricated 3D nanostructure as shown in Figure 4 (d), stairlike 3D nanostructure with six layers were successfully produced with clear height differences between adjacent steps. Depth of each layer is plotted in Figure 4 (e). The measuring direction is from the top across to the bottom aligning with the direction of the step. The depth of each step is designed to increase by $10 \mathrm{~nm}$. Due to the noise and other factors, there are some height variations along each step. The surface roughnesses of each step from the first layer to the sixth layer are 3.2, 2.4, 2.4, 2.9, 3.2 and 3.7 $\mathrm{nm}$. There is some residue debris after cleaning for the last step of the stair-like nanostructure, which results in a relative larger surface roughness. The depths of last two layers gradually become shallower when measuring from the top to the bottom. The reason may be imperfect ultrasonic cleaning process that 
did not thoroughly remove all the debris covering on the features. Another reason may be the interaction of the AFM tip and the debris prevents the right tip-sample engagement, since the machining process started from the top and finished at the bottom, which accumulates more debris at the bottom area. The widths of each stair are $250 \mathrm{~nm}$, which are the same as the designed values.

The layer-by-layer machining maintains the constant setpoint force to achieve the same depth during machining processes for each layer. The feature on each layer is machined by the constant depth, which makes it easy to control the process and compromises the setpoint force control capabilities from the AFM system. The limitation of the layer-by-layer machining process comes from its relatively complex machining procedure and the resulting low machining speed. Multiple images have to be designed and applied for the machining of a single structure, which can result in a low machining efficiency, especially for a large area. Moreover, the rough machined surface is the source of the disturbances for the setpoint force control system, which may further degrade the surface finish of the following layers. As a result, the area that is machined multiple times tends to have a larger surface roughness.

Besides the 3D nanofabrication using layer-by-layer machining, one pass nanomachining with depth regulated by the setpoint force is also investigated in the raster scan mode. A similar stair-like 3D nanostructure with 6 height levels was designed by a bitmap image with six different gray-scale, with each gray-scale representing a setpoint force value, and then the machining depth, as shown in Figure 5 (a). The designed bitmap image is given in the Figure 5 (e). The number of pixels for this image is selected to be $150 \times 150$. The overall size for the designed 3D nanostructure is $1.6 \times 1.6 \mu \mathrm{m}$ with the width of each stair about $265 \mathrm{~nm}$. The setpoint forces corresponding to the six gray-scale are 150, 180, 210, 240, 270 and $300 \mathrm{nN}$ from the darkest color to the brightest color. To machine this structure, the scanning speed is selected to be $1 \mu \mathrm{m} / \mathrm{s}$, the driving voltages of xy-vibration and z-vibration to be $30 \mathrm{mV}$ and $3 \mathrm{~V}$ respectively. The image and the 3D view of the fabricated structure are shown in Figure 5 (b) and (c). Figure 5 (d) shows the stair-like 3D nanostructure with six layers were successfully machined on the PMMA surface with the depth for each layer of $9 \mathrm{~nm}$. Figure 5 (f) shows the cross-sectional profile of each layer of the stair-like 3D nanostructure. The depths of each step is designed to be $8 \mathrm{~nm}$. Height variations is observed from the experiment along each step in the nanostructure. The surface roughness of each step from the first layer to the sixth layer are $0.8,1.0,1.3,0.9,1.1$ and $1.9 \mathrm{~nm}$. The depth at the left side of each step is shallower than that of the right side, especially when the setpoint force is large. The reason may be that the machining speed is a little high that the AFM tip is not able to indent deep enough before continue moving toward to the right side. Another reason may come from the slow setpoint force control systems of the AFM, in which the setpoint force is not instantly approach to the designed value when machining begins from the left side. 
(a)

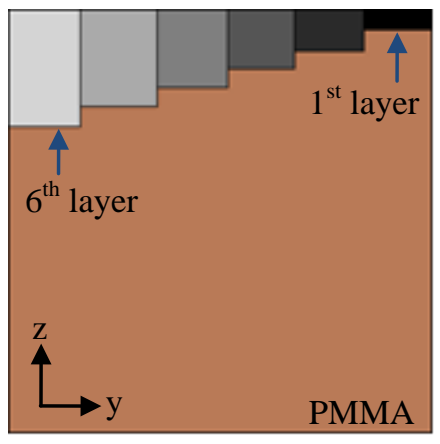

(b)

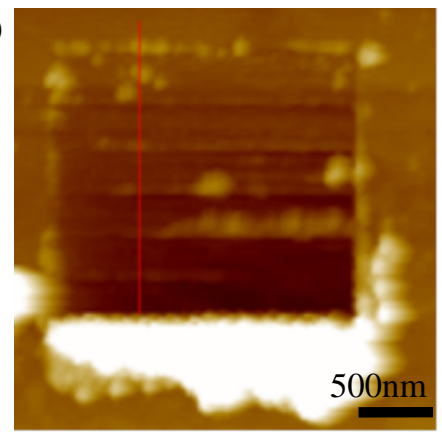

(c)

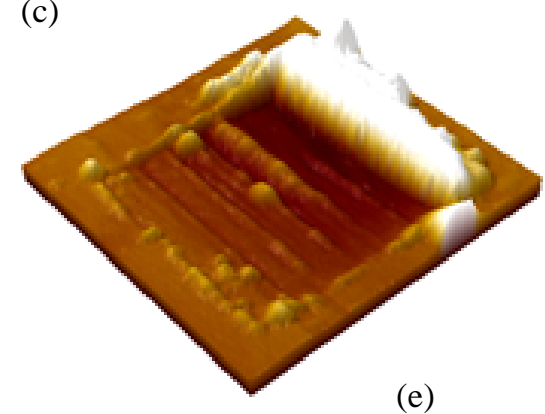

(d)
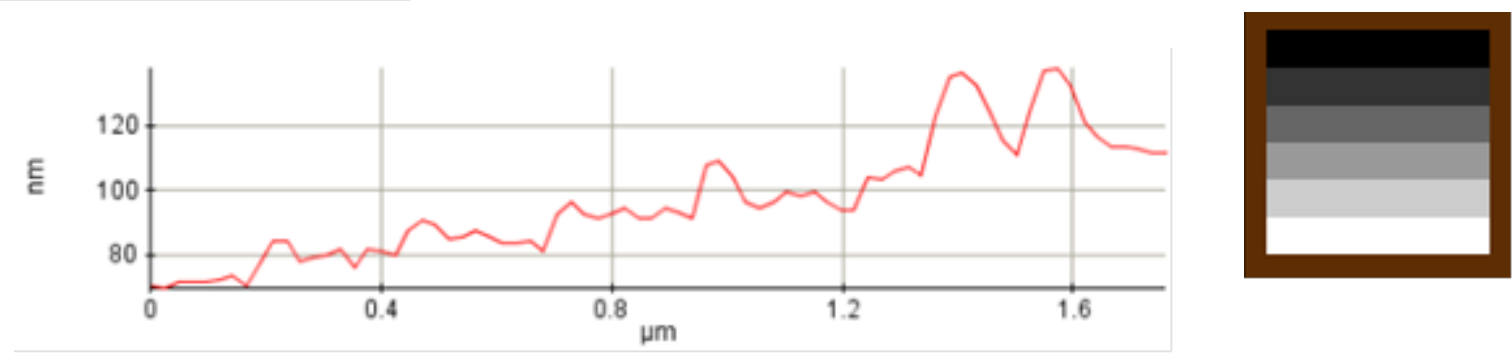

(f)

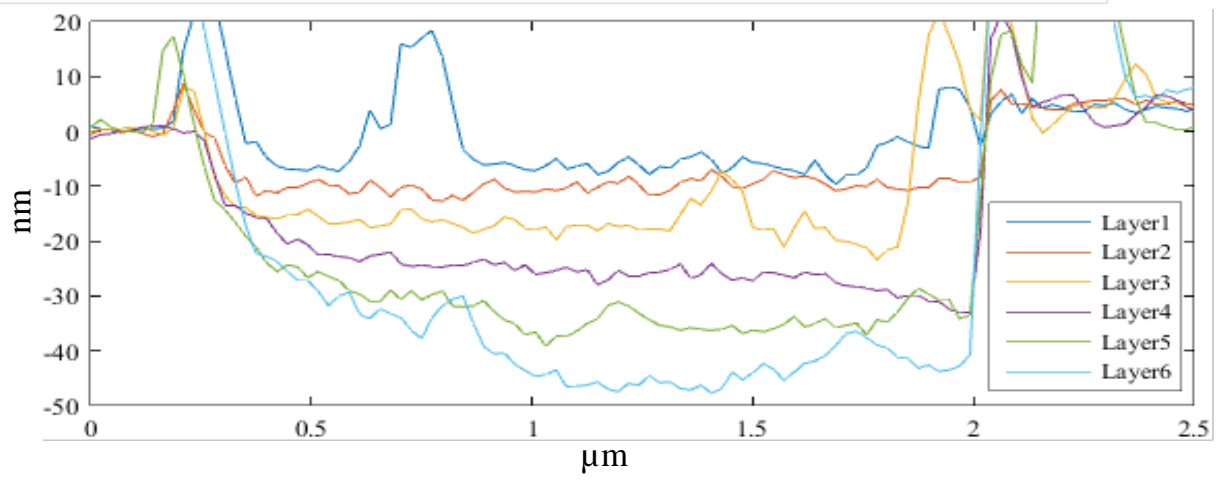

Figure 5: (a) Schematic of one pass nanomachining with depth regulated by the setpoint force in raster scan mode. (b) AFM image of the fabricated 3D nanostructure. (c) 3D view of the nanostructure. (d) Cross sectional profile shows 6 steps of the nanostructure. (e) Bitmap images designed for the 6 layers nanostructure. (f) Crosssectional profile of each steps of 6 layers.

Comparing the results fabricated by layer-by-layer machining and one pass machining, both of the two fabrication processes can be successfully used to fabricate multi-layer nanostructures. Machining parameters used in both process are similar to each other. One pass machining is very easy to implement with one gray-scale bitmap image. The alignment error from aligning multiple images in layer-by-layer machining is not a problem in one pass machining, as only one image is used. The disadvantage of the one pass machining is the difficulty in precisely control the feature dimension, as the gray-scale value directly represents the setpoint force, not the feature depth. The relationship between the setpoint force and the feature depth needs to be characterized experimentally, and in many cases may not be linear across a large force range. The response time of the setpoint force change from the AFM control system could be another limitation, especially when the setpoint force changes quickly and frequently from very complex part design. 


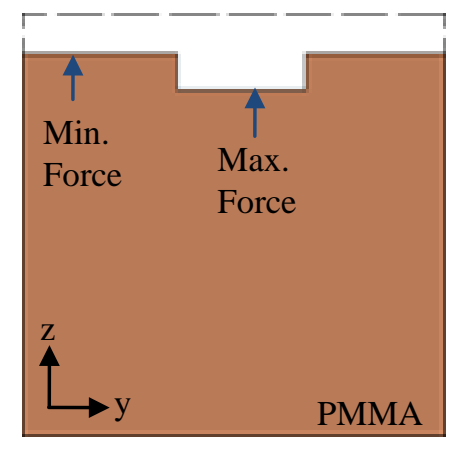

(b)
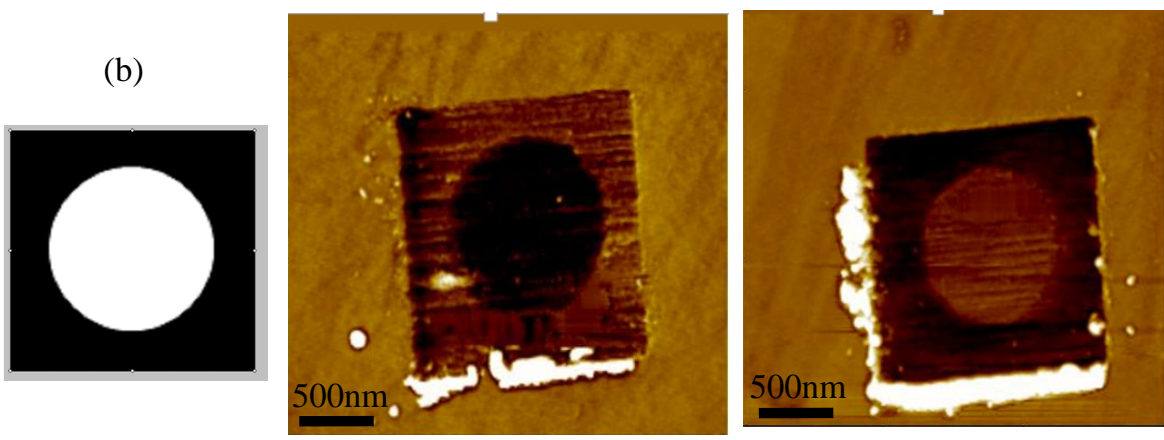

Figure 6: (a)Schematic of force applied according to the gray-scale in (b). (b) Bitmap images for the structure with a concave circle in a square. (c, d) Fabricated nanostructure from bitmap images.

Other 3D nanostructures were also fabricated to further demonstrate the capability of this one pass machining with force regulation in the raster scan mode. A concave circle in a square was designed as the bitmap image shown in Figure 6 (b). The resulting force applied on the sample surface according to the gray-scale is shown in Figure 6 (a). White and black regions of the image correspond to the maximum setpoint force of $150 \mathrm{nN}$ and minimum setpoint force of $5 \mathrm{nN}$ respectively. Setpoint forces applied on sample surfaces is dynamically changed when the tip scans across pixels with different gray-scale values. The result after fabrication, corresponding to the bitmap image of a concave circle pattern is shown in Figure 6 (c). Similarly, a structure with a convex circle in a square was fabricated by simply inverting the white and black colors in the bitmap image, with the fabricated nanostructure shown in Figure 6 (d). The fabricated results replicated the patterns from the bitmap image very well, which demonstrate the capability of 3D nanofabrication by regulating setpoint force applied on samples in the raster scan mode.

\section{Conclusions}

In this paper, we developed an AFM-based 3D nanofabrication process is developed using ultrasonic vibration assisted nanomachining. Two pattern design modes were utilized in this nanomachining process, which are vector mode based pattern generation and raster scan mode based pattern generation. The 3D nanostructures fabricated under vector mode and raster scan mode demonstrated that they are capable to fabricate 3D nanostructures using the novel ultrasonic assisted nanomachining processes. The critical process parameters are identified to be the setpoint force, the overlap percentage, amplitude of $\mathrm{z}$ vibration and machining speed. These parameters in the process needs be tuned carefully to achieve the desired feature dimension and process performance. While the vector mode is very flexible and efficient to create sparse patterns, raster scan mode can conveniently fabricate 3D nanostructures in one pass with the feature depth regulated by the force specified by the gray-scale bitmap image. The experimental fabrication results demonstrate the 3D nanofabrication capability of the AFM-based ultrasonic vibration assisted nanomachining process. 


\section{Acknowledgement}

This work was supported in part by the National Science Foundation under Grant Award NSF CMMI-1233176.

\section{References}

[1] Rubio - Sierra, F. J., Heckl, W. M., \& Stark, R. W. (2005). Nanomanipulation by atomic force microscopy. Advanced Engineering Materials, 7(4), 193-196.

[2] Rosa, J. C., Wendel, M., Lorenz, H., Kotthaus, J. P., Thomas, M., \& Kroemer, H. (1998). Direct patterning of surface quantum wells with an atomic force microscope. Applied Physics Letters, 73(18), 2684-2686.

[3] Fang, T. H., \& Chang, W. J. (2003). Effects of AFM-based nanomachining process on aluminum surface. Journal of Physics and Chemistry of Solids, 64(6), 913-918.

[4] Firtel, M., Henderson, G., \& Sokolov, I. (2004). Nanosurgery: observation of peptidoglycan strands in Lactobacillus helveticus cell walls. Ultramicroscopy, 101(2), 105-109.

[5] Wen, C. K., \& Goh, M. C. (2004). AFM nanodissection reveals internal structural details of single collagen fibrils. Nano Letters, 4(1), 129-132.

[6] Piner, R. D., Zhu, J., Xu, F., Hong, S., \& Mirkin, C. A. (1999). "Dip-pen" nanolithography. Science, 283(5402), 661-663.

[7] Martinez, J., Martínez, R. V., \& Garcia, R. (2008). Silicon nanowire transistors with a channel width of $4 \mathrm{~nm}$ fabricated by atomic force microscope nanolithography. Nano Letters, 8(11), 36363639.

[8] Martín, C., Rius, G., Borrisé, X., \& Pérez-Murano, F. (2005). Nanolithography on thin layers of PMMA using atomic force microscopy. Nanotechnology, 16(8), 1016.

[9] Wendel, M., Lorenz, H., \& Kotthaus, J. P. (1995). Sharpened electron beam deposited tips for high resolution atomic force microscope lithography and imaging. Applied Physics Letters, 67(25), 3732-3734.

[10] Yan, Y., Sun, T., Liang, Y., \& Dong, S. (2007). Investigation on AFM-based micro/nano-CNC machining system. International Journal of Machine Tools and Manufacture, 47(11), 1651-1659. 
[11] Göbel, H., \& Von Blanckenhagen, P. (1995). Atomic force microscope as a tool for metal surface modifications. Journal of Vacuum Science \& Technology B, 13(3), 1247-1251.

[12] Li, X., Wang, X., Xiong, Q., \& Eklund, P. C. (2005). Top-down structure and device fabrication using in situ nanomachining. Applied Physics Letters, 87(23), 233113.

[13] Yan, Y., Hu, Z., Zhao, X., Sun, T., Dong, S., \& Li, X. (2010). Top - Down Nanomechanical Machining of Three - Dimensional Nanostructures by Atomic Force Microscopy. Small, 6(6), 724-728.

[14] Hu, S., Altmeyer, S., Hamidi, A., Spangenberg, B., \& Kurz, H. (1998). Novel approach to atomic force lithography. Journal of Vacuum Science \& Technology B, 16(4), 1983-1986.

[15] Gozen, B. A., \& Ozdoganlar, O. B. (2010). A rotating-tip-based mechanical nano-manufacturing process: nanomilling. Nanoscale Research Letters, 5(9), 1403-1407.

[16] Schmid, S. R., \& Hector, L. G. (1998). Simulation of asperity plowing in an atomic force microscope. Part II: plowing of aluminum alloys. Wear, 215(1), 257-266.

[17] Liu, X., \& Zhang, H. (2007). Scanning probe microscopy-based nanofabrication for emerging applications. Modern Research and Educational Topics in Microscopy, Microscopy Series, (3), 770-778.

[18] Yamazaki, K., Yamaguchi, T., \& Namatsu, H. (2004). Three-dimensional nanofabrication with 10nm resolution. Japanese Journal of Applied Physics, 43(8B), L1111.

[19] Li, L., Hong, M., Schmidt, M., Zhong, M., Malshe, A., Huis in’tVeld, B., \& Kovalenko, V. (2011). Laser nano-manufacturing-state of the art and challenges. CIRP Annals-Manufacturing Technology, 60(2), 735-755.

[20] Ali, M., Wagner, T., Shakoor, M., \& Molian, P. A. (2008). Review of laser nanomachining. Journal of Laser Applications, 20(3), 169-184.

[21] Nellen, P. M., Callegari, V., \& Brönnimann, R. (2006). FIB-milling of photonic structures and sputtering simulation. Microelectronic Engineering, 83(4), 1805-1808.

[22] Lee, K. S., Kim, R. H., Yang, D. Y., \& Park, S. H. (2008). Advances in 3D nano/microfabrication using two-photon initiated polymerization. Progress in Polymer Science, 33(6), 631-681.

[23] Yang, S. M., Jang, S. G., Choi, D. G., Kim, S., \& Yu, H. K. (2006). Nanomachining by colloidal lithography. Small, 2(4), 458-475. 
[24] Berenschot, E. J., Jansen, H. V., \& Tas, N. R. (2013). Fabrication of 3D fractal structures using nanoscale anisotropic etching of single crystalline silicon. Journal of Micromechanics and Microengineering, 23(5), 055024.

[25] Berenschot, E., Tas, N. R., Jansen, H. V., \& Elwenspoek, M. (2008, January). 3D-nanomachining using corner lithography. In Nano/Micro Engineered and Molecular Systems, 2008. NEMS 2008. 3rd IEEE International Conference on (pp. 729-732). IEEE.

[26] Zhang, L., \& Dong, J. (2012). High-rate tunable ultrasonic force regulated nanomachining lithography with an atomic force microscope. Nanotechnology, 23(8), 085303.

[27] Zhang, L., Dong, J., \& Cohen, P. H. (2013). Material-insensitive feature depth control and machining force reduction by ultrasonic vibration in AFM-based nanomachining. IEEE Transactions on Nanotechnology, 12(5), 743-750. 